Ann. Sci. forest., 1978, 35 (4), 237-263.

\title{
Variabilité infraspécifique du retrait avec collapse et de la densité du bois chez Eucalyptus camaldulensis
}

\author{
A. SESBOU ef G, NEPVEU * \\ Ecole nationale forestière d'Ingénieurs, Sale (Maroc) \\ * Station de Recherches sur la Qualité des Bois, \\ Centre national de Recherches forestières, I.N.R.A. \\ Champenoux, 54280 Seichomps
}

\section{Résumé}

\begin{abstract}
A partir de deux dispositifs situés en Italie (Sicile et Calabre), vingt-quatre provenances d'Eucalyptus camaldulensis ont été étudiées du point de vue des retraits avec collapse et de la densité de leur bois. Ces caractères ont été mesurés sur carottes de sondage ( 2400 échantillons).
\end{abstract}

Les principaux résultats sont les suivants :

- les valeurs moyennes atteintes pour les retraits avec collapse sont considérables : 0,72 p. 100 pour le retrait axial, 28 p. 100 pour le retrait tangentiel, 7,8 p. 100 pour le retrait radial, 39 p. 100 pour le retrait volumétrique. Un reconditionnement à la vapeur d'eau permet foutefois de récupérer 46 à 74 p. 100 du retrait tangentiel;

- les variabilités individuelle et infraspécifique sont très importantes pour les retraits et l'infradensité. Le retrait volumétrique peut varier suivant les provenances de 18 p. 100 à 58 p. 100, la densité de 443 à $593 \mathrm{~g} / \mathrm{dm}^{3}$ :

- une grande région de provenances, homogène, le bassin de la rivière Murray, a èté mise en évidence ; elle se caractérise par des densités faibles, une croissance ef des retraits élevés ;

- les liaisons aux niveaux individuel et infraspécifique sont négatives entre retraits et densité. Entre moyennes de provenances, les corrélations vigueur-densité sont négatives, positives entre vigueur et retrait :

- la réputation calcifuge de $E$. camaldulensis semble remise en cause par les bons résultats observés en Calabre sur la vigueur. En plus d'une meilleure croissance, les arbres présentent dans cette station une densité plus élevée, un retrait plus faible qu'en Sicile :

- les interactions génotype-environnement semblent faibles pour les caractères étudiés.

\section{1. - Introduction.}

Le genre Eucalyptus, myrtacée originaire d'Australie, comprend plus de sept cents espèces ou variétés dont le nombre augmente chaque année à la suife d'hybridations, tant naturelles qu'artificielles.

La première Conférence Mondiale sur l'Eucalyptus, qui s'est tenue à Rome en 1956. a mis en relief l'importance mondiale acquise par ce genre ; elle est à attribuer, d'une part à la pénurie de bois en quantité ef en qualité dans certaines régions du monde, et d'autre part à la plasticité des Eucalyptus, ce qui les rend extrêmement intéressants dans des sites très nombreux.

Mais on a constaté que le développement de l'utilisation de son bois, dans d'autres pays que l'Australie, n'avait pas en général suivi le prog rès de la culture du genre. 
Parmi les Eucalyptus, le camaldulensis connait la plus grande utilisation. Les généticiens se sont attachés avant tout à l'améliorer dans le but d'assurer la satisfaction des besoins quantitatifs en matière ligneuse des pays concernés.

Or des études récentes, conduites sur de nombreuses espèces, ont montré l'intérêt de la sélection pour la qualité. Ceci peut s'avérer très intéressant dans le cas du camaldulensis qui, en effet, est connu par certains défauts assez graves qui ont amené jusqu'à présent à l'éliminer des utilisations nobles.

La présente étude a précisément pour but d'apprécier la variabilité infraspécifique de l'Eucalyptus camaldulensis pour certaines propriétés physiques conditionnant largement son emploi industriel. En plus de l'intérêt économique mis en évidence ci-dessus, nous soulignerons la qualité des dispositifs sur lesquels nous avons travaillé, ce qui, à notre sens, donne une portée assez large aux résultats obtenus.

En premier lieu, nous donnerons quelques indications sur les dispositifs étudiés, les caractères pris en considération, leur intérêt et les méthodes mises en œuvre pour les apprécier.

Dans une seconde partie, nous nous pencherons sur les variabilités individuelles et infraspécifiques des critères qui nous ont intéressés, leurs relations entre eux et avec la vigueur. Nous analyserons l'importance éventuelle des corrélations génotypeenvironnement.

\section{2. - Matériel et méthode}

\section{1. - Matériel}

Compte tenu de l'influence des facteurs écologiques et édaphiques sur les caractéristiques physiques et mécaniques du bois (Polge, 1973), des possibilités d'existence d'interactions génotype - environnement, il nous a semblé intéressant de prélever les échantillons sur deux stations appartenant à des milieux différents.

Ces dispositifs font partie du projet no 6 du Comité de la Recherche Forestière Méditerranéenne de la FAO intitulé : «Etude de l'adaptation écologique des Eucalyptus » qui s'est concrétisé par l'installation de trente et une plantations comparatives de provenances d'Eucalyptus camaldulensis réparties entre douze pays (Algérie, Chypre, Congo, Espagne, Grèce, Israël, Italie, Maroc, Nigéria, Portugal, Tunisie, Turquie). Son but essentiel consistait à promouvoir la production de bois d'industrie dans des pays en voie de développement de la zone méditerranéenne. Notre étude déborde un peu de cet objectif puisque nous avons pris en considération des caractères - retraits du bois - importants pour un usage essentiellement noble du matériau ligneux. On se reportera avec intérêt au rapport de son coordonnateur : Lacaze (1970) qui en analyse les premiers résultats en ce qui concerne les caractères de vigueur.

\subsection{Caractéristiques des stations.}

Elles sont présentées dans le tableau 1.

\subsection{Provenances utilisées.}

Pour les deux stations, on a utilisé vingt-quatre provenances dont une, la 7116 a été employée deux fois pour satisfaire ainsi aux contraintes du dispositif. 
TABLEAU 1

Caractéristiques des plantations comparatives (Characteristics of provenance plantations)

Station 1 : Sicile

Caltagirone (Terrana)

$232 \mathrm{~m}$

4 ha

$500 \mathrm{~mm}$

$17,8^{\circ} \mathrm{C}$

$5,9^{\circ} \mathrm{C}$

$32,8^{\circ} \mathrm{C}$

63,6

Semi-aride supérieur à variante tempérée

Sol sablo-argileux; sous-sol compact assez argileux : $\mathrm{pH}$ neutre à largement acide (absence de calcaire)

Subéraie dégradée avec abondante végétation arbustive de lentisque, phylleria et cistes

Automne 1966

En plein sur $50 \mathrm{~cm}$ de profondeur après élimination des souches par des moyens mécaniques

24 janvier 1967

$3 \mathrm{~m} \times 3 \mathrm{~m}$

25
Station 2 : Calabre

Catanzano (ferme Condo-

léo)

$20 \mathrm{~m}$

5 ha

$600 \mathrm{~mm}$

$17,5 \circ \mathrm{C}$

$5,3 \circ \mathrm{C}$

$32,10 \mathrm{C}$

77,0

Subhumide inférieur à variante tempérée

Régosol limono-argileux superficiellement : soussol argilo-limoneux avec drainage insuffisant ; $\mathrm{pH}$ légèrement supérieur à 7 avec présence de calcaire

Pâturage broussailleux avec lentisque et oléastre

\section{Eté 1966}

En plein sur $50 \mathrm{~cm}$ de profondeur

15 mars 1967

$3 \mathrm{~m} \times 3 \mathrm{~m}$

25

Elles appartiennent aux quatre grandes régions de l'aire de l'Eucalyptus camaldulensis :

- Territoire du Nord (Northern Territory) avec cinq provenances : 6869,7080 , $7116 \mathrm{~A}, 7116 \mathrm{~B}$ et 6788 ;

- Australie Occidentale(Western Australia) avec deux provenances : 7029 ef 7049 ;

- Bassin de la rivière Murray et Australie Méridionale (Murray River System) 
TABLEAU 2

Origine des provenances (Origin of provenances)

\begin{tabular}{|c|c|c|c|c|c|c|}
\hline No & Nom (Name) & $\begin{array}{l}\text { Sous-région } \\
\text { (Subregion) }\end{array}$ & Région (Region) & $\begin{array}{c}\text { Altitude } \\
\text { (Altitude) } \\
\text { (m) }\end{array}$ & $\begin{array}{l}\text { Pluviométrie } \\
\text { (Precipitation) } \\
(\mathrm{mm})\end{array}$ & $\begin{array}{l}\text { Saison sèche } \\
\text { (Dry season) }\end{array}$ \\
\hline $1=6788$ & Todd River, Alice Springs & Sud & Territoire du Nord (NT) & 579 & 246 & hiver \\
\hline $2=6843$ & Sherppaton (V) & Rivière Murray & $\begin{array}{l}\text { Bassin de la rivière } \\
\text { Murray }\end{array}$ & - & - & été \\
\hline $3=6844$ & Woohlpooer State Forest (V) & Type de plaine & $x$ & 300 & - & été \\
\hline $4=6845$ & Lake Albacutya (V) & Lac Salé & s & 183 & 341 & été \\
\hline $5=6869$ & Katherine & Nord & Territoire du Nord & 110 & 943 & hiver \\
\hline $6=6870$ & Eulo (Q) & R. Paroo & Australie méridionale & 183 & 192 & été \\
\hline $7=6948$ & $\begin{array}{l}\text { Bullock Creek (Q) } 64 \mathrm{~km} \text { est de Hu- } \\
\text { ghenden }\end{array}$ & Bassin du Lac Eyre & Quennsland Nord & 457 & 456 & hiver \\
\hline $8=6953$ & Petford (Q) & $\begin{array}{l}\text { Affinité avec E. Tereti- } \\
\text { cornis }\end{array}$ & s & 510 & 704 & hiver \\
\hline $9=6958$ & Bourke (NSW) & R. Darling & Australie méridionale & 110 & 904 & étè \\
\hline $10=6966$ & Est d'Adelaïde Menger's Hill (SA) & Hill sife & $\begin{array}{l}\text { Bassin de la rivière } \\
\text { Murray }\end{array}$ & 600 & 1163 & éfé \\
\hline $11=6968$ & s & s & m & n & s & b \\
\hline $12=6969$ & 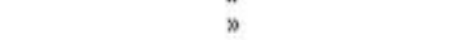 & 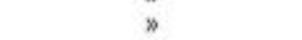 & is & o & w & 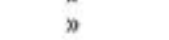 \\
\hline $13=6970$ & s & 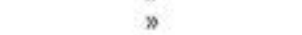 & s & ” & s & s \\
\hline $14=6971$ & s & s & s & n & " & s \\
\hline $15=6973$ & ) & ) & $\mathrm{s}$ & n & s & ) \\
\hline $16=6974$ & s & s & s & o & is & b \\
\hline $17=6975$ & Port Lincoln Little Swamp (SA) & Sol calcaire & 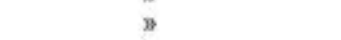 & 30 & 456 & été \\
\hline $18=6978$ & ( & 皮 & n & $x$ & s & 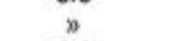 \\
\hline $19=6990$ & Darlington Point (NSW) & R. Murrumbidgee & Australie méridionale & 91 & 379 & été \\
\hline $20=6991$ & Walpola island St Forest (NSW) & Rivière Murray & $\begin{array}{l}\text { Bassin de la rivière } \\
\text { Murray }\end{array}$ & & 243 & êté \\
\hline $21=7029$ & Murchinson River & s & Australie occidentale & 213 & 465 & èté \\
\hline $22=7046$ & Wiluna-Gum Creek & Northern Gold Fields & o & 480 & 245 & été \\
\hline $23=7080$ & Newcastle Waters Creek & Nord & Territoire du Nord & 213 & 475 & hiver \\
\hline $24=7116 \mathrm{~A}$ & Tennant Creek, Goodairs Creek & Sud & $x$ & - & - & - \\
\hline $25=7116 B$ & (a) & » & s & - & - & - \\
\hline
\end{tabular}


avec seize provenances : $6843,6844,6845,6870,6958,6990,6991,6966,6968,6969$, $6970,6971,6973,6974,6975$ et 6979 ; et 6953 .

- Queensland du Nord (Northern Queensland) avec deux provenances : 6948

Ces quatre grandes unités géographiques sont divisées en sous-régions de provenances.

Pour faciliter le traitement des données, la numérotation à quatre chiffres des provenances a été remplacée par une numérotation simple de 1 à 25 ; le tableau 2 donne l'équivalence entre les différents numéros ainsi que la localisation des origines dans les sous-régions. On se reportera également à la figure 1.

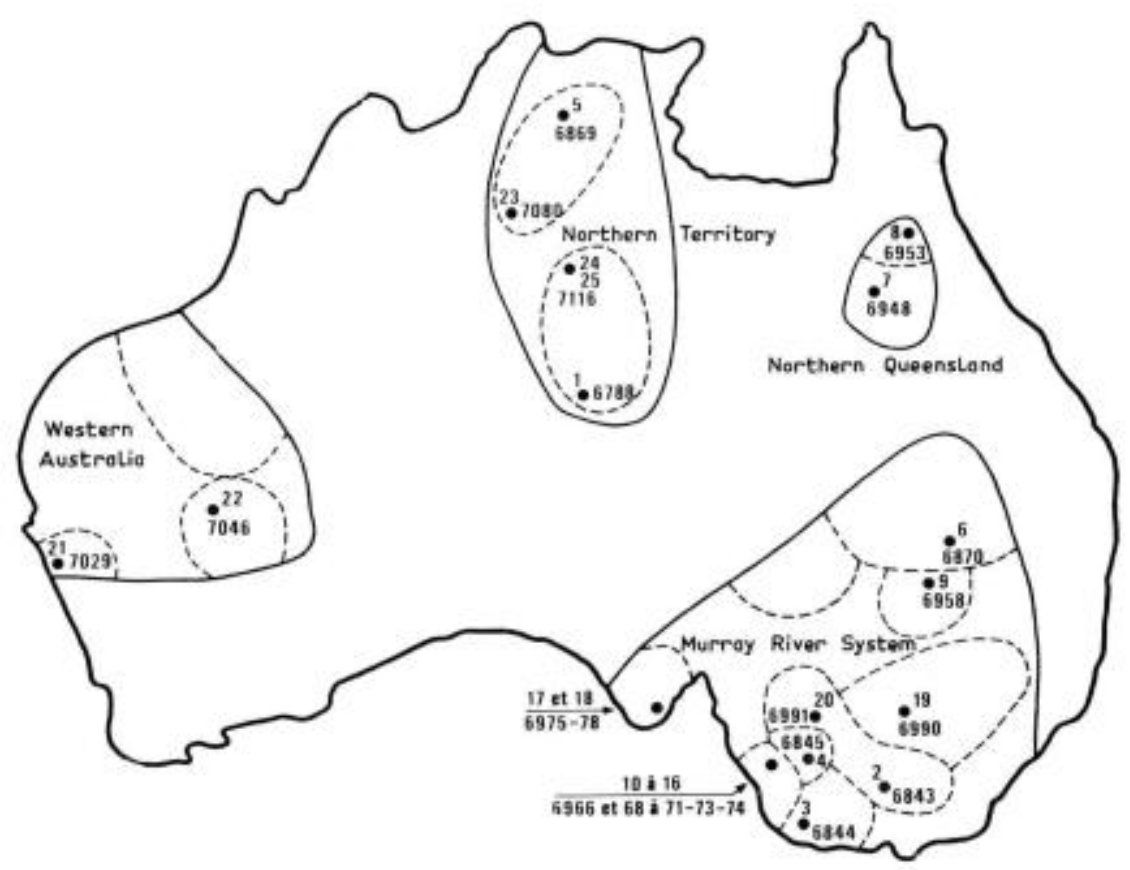

FIG, 1. - Localisation des provenances utilisées dans les expériences itoliennes. Location of the provenances in the fialion fests.

\subsection{Dispositif statistique.}

Le même dispositif est adopté pour les deux stations. II s'agit de blocs incomplets équilibrés dont les caractéristiques sont les suivantes:

- nombre de répétitions : 6 ;

- nombre de provenances : 25 (voir chapitre 2.12) ;

- nombre de blocs par répétition : 5 ;

- nombre de parcelles unitaires par bloc : 5 ;

- nombre d'arbres par parcelle unitaire : 25 . 


\subsection{Choix des arbres ef prélèverment des caroftes.}

La récolte a été effectuée au cours d'une mission en Italie du 14 au 19 décembre 1976. Dans chaque parcelle unitaire, deux arbres ont été choisis au hasard. Sur chaque arbre, deux carottes ont été prises à la tarière de Pressler entre $1 \mathrm{~m}$ et 1,50 $\mathrm{m}$ de hauteur dans le but d'apprécier la variabilité intra-arbre et afin de disposer d'un matériel suffisant pour les essais divers que nous prévoyons après cette étude. Après l'extraction de la carotte, les trous sont rebouchés à l'aide de chevilles traitées avec un fongicide destiné à empêcher l'attaque des arbres sondés par les parasites (Thiercelin et Polge, 1970 ; Thiercelin, Arnoud, Mangenot ef Polge, 1972).

Les éprouvettes traversent l'arbre d'un bout à l'autre, en passant le plus près du cœur. La direction des prélèvements est constante dans chacun des deux dispositifs, ce dans le but de fenir compte d'une éventuelle anisotropie systématique observée par de nombreux auteurs sur plusieurs essences (Polge et Illy, 1967 ; Janin, 1973 ; Nepveu, 1973 et 1976 ; Thoby, 1975 ; Nepveu, Keller et Teissier Du Cros, 1978).

Pour la station de Sicile, on a utilisé exclusivement des tarières de $5 \mathrm{~mm}$ de diamètre ; en Calabre, on a été contraint, à la suite du bris de deux d'entre elles, de recourir à un outil de $4 \mathrm{~mm}$.

Les carottes ont été conservées dans une solution de merseptyl à 1 p. 1000 pour éviter des attaques de champignons. Compte tenu de la longue période séparant la collecte des mesures ( 6 mois pour la Sicile), les échantillons provenant de Sicile ont été placés en chambre froide à $+4^{\circ} \mathrm{C}$ jusqu'au mois de juin.

II est un peu regrettable de n'avoir pas mélangé l'ensemble des éprouvettes recueillies car il n'est pas impossible, a priori, qu'un long séjour dans l'eau puisse modifier les caractéristiques du bois que nous avons mesurées ; ceci pourra faire apparaître artificiellement un effet «station ".

\section{2. - Description des essais}

Les essais effectués ont porté sur le retrait et l'infradensité. Ils ont été réalisés à la Station de Recherches sur la Qualité des Bois du Centre National de Recherches Forestières.

Avant d'entrer dans des considérations d'ordre méthodologique, nous attirerons l'attention du lecteur sur les deux phénomènes pouvant accompagner le séchage des bois, en particulier ceux d'Eucalyptus, à savoir le retrait et le collapse.

\subsection{Quelques rappels sur le refrait et le collapse.}

\subsection{Définition du retrait.}

II s'agit de l'ensemble des phénomènes qui accompagnent les variations d'humidité dans le bois. Ils se manifestent par des variations dimensionnelles qui peuvent être accompagnées de déformations et de fentes. Cette propriété caractéristique du bois permet de mesurer et d'expliquer les changements de dimensions du matériau au cours du séchage. Le bois étant anisotrope, les variations ne se font pas dans les mêmes proportions suivant les directions; on distingue dans une pièce un retrait axial dans le sens des fibres, un retrait radial dans le sens des rayons ligneux et un retrait tangentiel qui se manifeste tangentiellement aux cernes. 


\subsection{Causes du retrait.}

Le retrait apparait dès que l'humidité du bois devient inférieure au point de saturation des fibres. Dans un bois gorgé d'eau, cette dernière se trouve dans les parois des cellules (eau liée) et dans leurs cavités (eau libre). Lors du séchage, c'est l'eau libre qui part la première et son départ n'entraîne aucun changement de dimensions. Une fois le point de saturation des fibres atteint, il ne reste plus que l'eau des parois. Si on continue le séchage, le retrait apparaît.

Chez certaines essences, dans le genre Eucalyptus notamment, le retrait est précédé d'un autre phénomène plus grave: le collapse.

\subsection{Définition du collapse.}

C'est un défaut du séchage qui consiste en une contraction considérable et irrégulière du bois, distincte du retrait normalement constaté. Les pièces prennent une forme plus ou moins ondulée. Parfois, il y a apparition de fentes internes, invisibles de l'extérieur. Il affecte de nombreuses essences et particulièrement le genre Eucalyptus. En France, si le phénomène est limité, « le chêne, le noyer, le hêtre et certaines races de peuplier seraient susceptibles de subir le phénomène » (Villière, 1966).

\subsection{Causes du collapse.}

Le collapse résulte du départ de l'eau libre. II se produit donc avant le retrait normal. Diverses hypothèses ont été émises pour expliquer ce phénomène:

D'après Tiemann (1915), les cellules qui s'effondrent doivent être complètement remplies d'eau sans aucune trace d'air. Durant un séchage rapide, l'eau passe d̀ travers les membranes et si l'air ne peut pas pénétrer, il se produit un vide très poussé qui développe des tensions susceptibles d'écraser les cellules internes.

Pour Stamm (1929), un bois humide exposé dans une atmosphère desséchante arrive à perdre son eau très rapidement en surface ; cette dernière est soumise, en conséquence, à des efforts de tension. Si ceux-ci ne sont pas compensés par des fentes, ils exercent une forte compression sur la partie interne du bois. A partir d'un certain seuil, les parois des fibres peuvent s'effondrer.

Des études faites au Japon (Kanagawa, 1977) ont montré qu'au cours d'un séchage à $20^{\circ} \mathrm{C}$, le collapse affecte d'abord les rayons ligneux et le parenchyme axial.

Notons que le bois provenant d'arbres jeunes est plus sensible au collapse que celui des arbres âgés (Elliott, 1930). Nous garderons ce fait à l'esprit en songeant que nous travaillons sur des sujets plantés depuis 10 ans.

2.215. Un moyen de réduire le collapse : le reconditionnement.

- But : Il a pour but de provoquer un ramollissement des parois cellulaires ; les éléments anatomiques peuvent alors, grâce à leur élasticité naturelle, reprendre plus ou moins leurs formes normales, d'où augmentation générale des dimensions du bois.

- Principe : On fait un séchage jusqu'à 15 p. 100 d'humidité ; ensuite les échanfillons sont passés à la vapeur à une certaine température et pendant un certain temps. Durant ce traitement, le bois peut résorber une légère quantité d'humidité ef il est nécessaire donc de le ressécher à nouveau. La récupération du collapse est irréversible, et il n'y a plus de risque d'observer ce phénomène dans un bois reconditionné. 
- Traitement des bois collapsés : Greenhill (1938) a fait de nombreux essais pour déterminer les meilleures conditions de traitement. Ses conclusions sont les suivantes :

- la récupération du collapse n'est jamais complète,

- avec des températures élevées, on parvient à une « récupération » maximum,

- plus la température du traitement est élevée, plus sa durée est faible,

- on obtient de bons résultats en reconditionnant à la vapeur à $200^{\circ} \mathrm{C}$ pendant 1 à $2 \mathrm{mn}$,

- on peut même améliorer le traitement en utilisant certains produits chimiques (chlorure de zinc, chlorure de sodium) (Kauman, 1960a ef 1960 b) dans le bois avant séchage : sous l'action de la chaleur, ils dégagent des gaz maintenant une pression intracellulaire après le départ de l'eau.

- Expériences de recondifionnement : Nous avons fait quelques expériences de reconditionnement de bois collapsé :

- Reconditionnement à l'eau bouillante : Deux échantillons d'Eucalyptus effondrés ont été mis à bouillir dans de l'eau pendant 48 h. Après séchage (10 p. 100 d'humidité), le retrait a été mesuré. Le tableau 3 donne les résultats obtenus :

TABLEAU 3

Résulfats du recondifionnement à l'eau bouillante Results of retreatment with boiling water

\begin{tabular}{cccccc}
\hline \hline \multirow{2}{*}{$\begin{array}{c}\text { Echantillons } \\
\text { (Samples) }\end{array}$} & \multicolumn{2}{c}{$\begin{array}{c}\text { Diamètres moyens tangentiels }(\mu) \\
\text { (Tangential mean diameters) }\end{array}$} & & \multicolumn{2}{c}{$\begin{array}{c}\text { Diamètres moyens axiaux }(\mu) \\
\text { (Axial mean diameters) }\end{array}$} \\
\cline { 2 - 3 } \cline { 5 - 6 } & $\begin{array}{c}\text { Avant } \\
\text { reconditionnement } \\
\text { (Before retreatment) }\end{array}$ & $\begin{array}{c}\text { Après } \\
\text { reconditionnement } \\
\text { (After retreatment) }\end{array}$ & & $\begin{array}{c}\text { Avant } \\
\text { reconditionnement } \\
\text { (Before retreatment) }\end{array}$ & $\begin{array}{c}\text { Après } \\
\text { reconditionnement } \\
\text { (After retreatment) }\end{array}$ \\
\hline 1 & 3590 & 3251 & & 4374 & 4328 \\
2 & 3646 & 3443 & 4366 & 4346 \\
\hline \hline
\end{tabular}

On note dans tous les cas une diminution du diamètre moyen des carottes, donc une aggravation du collapse. Ceci est confirmé par Hayashi et Terrazawa (1977) qui ont montré que des bois bouillis collapsent même avec des basses températures de séchage.

- Reconditionnement à la vapeur : Dix carottes très collapsées ont été traitées à la vapeur à $130^{\circ} \mathrm{C}$ pendant $10 \mathrm{mn}$. Après séchage, le retrait tangentiel a été calculé. Les résultats obtenus montrent que ce procédé permet de récupérer une grande partie du collapse (tableau 4) et que le collapse est le principal responsable des variations dimensionnelles des échantillons qui nous intéressent, séchés sans précaution particulière.

II est intéressant de noter qu'il ne semble pas exister de liaisons entre les retraits avant ef après reconditionnement ( $r=0,246 \mathrm{NS})$.

\subsection{Mesure du retrait avec collapse.}

Le retrait a été mesuré sur les carottes suivant les trois directions : tangentielle, axiale, radiale, et entre les deux états successifs : 
TABLEAU 4

Résulfats du reconditionnement à la vapeur

Results of retreatment with steam

\begin{tabular}{|c|c|c|c|c|}
\hline $\begin{array}{l}\text { Echan- } \\
\text { tillons }\end{array}$ & $\begin{array}{l}\text { Avant recon- } \\
\text { ditionnement } \\
\text { p. } 100 \\
\text { (Before } \\
\text { retreatment) }\end{array}$ & $\begin{array}{l}\text { Après recon- } \\
\text { ditionnement } \\
\text { p. } 100 \\
\text { (After } \\
\text { retreatment) }\end{array}$ & $\begin{array}{c}\text { Gains en valeur absolue } \\
\text { p. } 100 \\
\text { (Gain in absolute value) }\end{array}$ & $\begin{array}{l}\text { gains en p. } 100 \text { du retrait } \\
\text { avant reconditionnement } \\
\text { (Gain in p. } 100 \text { of shrinkage } \\
\text { before retreatment) }\end{array}$ \\
\hline 1 & 35,61 & 9,32 & 26,29 & 74 \\
\hline 2 & 26,14 & 9,45 & 16,69 & 64 \\
\hline 3 & 35,23 & 9,43 & 26,00 & 74 \\
\hline 4 & 20,94 & 9,40 & 11,54 & 55 \\
\hline 5 & 23,09 & 8,67 & 14,42 & 62 \\
\hline 6 & 26,75 & 7,71 & 19.04 & 71 \\
\hline 7 & 26,26 & 14,12 & 12,14 & 46 \\
\hline 8 & 30,63 & 8,65 & 21,98 & 72 \\
\hline 9 & 42,11 & 12.74 & 29,37 & 70 \\
\hline 10 & 32,76 & 8,45 & 24,31 & 74 \\
\hline
\end{tabular}

- l'état saturé qu'on obtient par immersion des carottes dans l'eau pendant au moins 10 jours :

— l'état sec à l'air, correspondant à une humidité de 10 p. 100 , obtenu en séchant les carottes pendant $48 \mathrm{~h}$ dans une étuve hygrométrique à $62 \mathrm{p}$. 100 d'humidité (thermomètre humide à $42^{\circ} \mathrm{C}$ ef thermomètre sec à $50^{\circ} \mathrm{C}$ ), les carottes étant placées dans des presses comportant des cannelures rectilignes de $5 \mathrm{~mm}$ de diamètre (les carottes sont placées de sorte que le fil du bois soit vertical), afin d'éviter les déformations des carottes dues à l'anisotropie des retraits tangentiel et radial.

Les essais ont porté sur l'ensemble des carottes (2 400 au total), chaque éprouvette étant divisée en deux pour tenir compte d'un effet « orientation » éventuel.

Nous soulignons le fait qu'en l'absence de tout reconditionnement, le retrait dont il s'agit ici intègre les phénomènes de collapse.

\subsection{Retrait tangentiel ef retrait axial.}

Ces deux retraits ont été mesurés à partir des dimensions moyennes axiales et tangentielles des carottes à l'état saturé et à l'état sec à l'air. Elles sont obtenues grâce à une chaine de mesures mise au point à la Station (Juino, 1977).

Les longueurs à mesurer ont été choisies en fonction de celles des carottes, de façon à avoir pour chaque échantillon une moyenne de soixante mesures au minimum. Pour les longueurs L variant entre 30 et $60 \mathrm{~mm}$, on a pris un pas de $0,5 \mathrm{~mm}$, $1 \mathrm{~mm}$ pour $\mathrm{L}$ supérieure à $60 \mathrm{~mm}$ et $0,25 \mathrm{~mm}$ pour $\mathrm{L}$ inférieure à $30 \mathrm{~mm}$. La moyenne des mesures à l'état saturé étant ds et celle à l'état sec à l'air étant $d_{10}$, le retrait en p. 100 est calculé par la formule :

$$
R=\frac{d_{8}-d_{10}}{d_{10}} \times 100 .
$$




\subsection{Retrait radial.}

Une fois les mesures des retraits axial et radial terminées, les deux extrémités des échantillons sont découpées de façon nette et perpendiculairement à l'axe de la carotte pour éviter les erreurs dans le calcul du retrait radial (nous n'avons cette fois qu'une seule mesure à chaque état). Les mesures sont effectuées à l'aide d'un micromètre au $1 / 100$ e de $\mathrm{mm}$ aux deux états : saturé et sec à l'air. Le retrait radial est calculé à l'aide de la formule :

$$
R_{r}=\frac{L_{s}-L_{10}}{L_{10}} \times 100
$$

avec $L_{5}=$ longueur de l'éprouvette à l'état saturé, $L_{10}=$ longueur de l'éprouvette à l'état sec à l'air.

\subsection{Retrait volumétrique.}

II se calcule à partir des trois retraits précédents (Juino, 1977 op. cit.).

\subsection{Mesure de l'infradensité.}

C'est le rapport entre la masse anhydre $P_{0}$ et le volume saturé $V_{8}$. Il s'agit d'une caractéristique intéressante à évaluer car elle est le rapport de deux quantités parfaitement définies.

La détermination d'un volume étant difficile, surtout dans le cas du bois, il a fallu trouver une méthode ne faisant intervenir aucun volume. C'est la méthode de saturation intégrale qui a été utilisée. Elle est basée sur le fait que la matière ligneuse a une densité constante et égale à 1,53 (Keylwerth, 1954); elle a l'avantage de ne faire intervenir que les poids anhydre et saturé.

Pour notre expérience, l'infradensité a été calculée pour la moitié des échantillons car il nous importait d'en réserver une partie pour la détermination des composantes de la densité ef la mesure des caractéristiques papetières. Nous avons donc retenu deux demi-carottes situées à des orientements et sur des éprouvettes différents.

Le poids maximum (Pmax) a été obtenu par saturation intégrale par ébullition pendant $48 \mathrm{~h}$ suivie d'une période de refroidissement. Le poids anhydre $\left(\mathrm{P}_{0}\right)$ a été obtenu après passage à l'étuve à $102,5^{\circ} \mathrm{C}$ pendant une durée minimum de $48 \mathrm{~h}$. Les pesées ont été faites avec une balance au $1.10^{-4} \mathrm{~g}$ reliée à une imprimante couplée à un perforateur de bandes.

Une fois les poids anhydre et saturé connus, une calculatrice permet, à l'aide d'un programme, de donner les valeurs correspondantes de l'infradensité.

\section{3. - Résultats et discussion}

\section{1. - Généralités}

Dans chacune des deux stations (Sicile et Calabre), un nombre important d'effets pouvait être éfudié :

- pour les retraits : les effets « provenance », « répétition », «bloc dans répétition », "arbre dans parcelle unitaire », «carotte dans arbre » et « demi-carotte dans carotte $\$$ (terme d'erreur) : 
- pour l'infradensité : les effets «provenance », « répétition », « bloc dans répéfition », « arbre dans parcelle unitaire », « demi-carotte dans arbre " (terme d'erreur incluant à la fois une variabilité due à l'orientement et, ce dernier facteur étant fixé, à la position sur le tronc le long d'une génératrice).

Afin de tenir compte de toutes ces sources de variation, les contraintes du calcul automatique nous ont obligés à procéder en deux temps :

Etude de la variabilité individuelle.

Les effets « provenance », « répétition » et « bloc dans répétition » ont été confondus en un effet "parcelle unitaire "; on a donc affaire à une analyse de variance hiérarchique oủ sont testés en plus de l'effet déjà cité, ceux de l'arbre, de l'échantillon dans l'arbre, et éventuellement de la demi-carotte dans la carotte (cas des retraits) Cefte étude est importante car elle permet de décomposer la variabilité intraprovenance, donc en définitive de juger de l'intérêt d'une sélection individuelle dans des origines intéressantes.

\section{Etude de la variabilité infraspécifique.}

Cette fois, on considère les huit demi-carottes (cas des retraits) ou les quatre demicarottes (cas de l'infradensité) par parcelle unitaire comme de simples répétitions. On éfudie les effets «provenance », « répétition » et «bloc dans provenance ».

\section{2. - Efude de la variabilité individuelle}

3.21. Etude de la variance.

Les analyses de variance hiérarchiques étaient de deux sortes suivant qu'il s'agissait des retraits ou de l'infradensité :

- Dans le premier cas, on avait selon les stations le tableau suivant:

TABLEAU 5

Décomposition de la variance pour les retraits

Partitioning of variance for shrinkage

\begin{tabular}{|c|c|c|c|c|}
\hline \multirow{2}{*}{$\begin{array}{l}\text { Sources de variation } \\
\text { (Sources of variation) }\end{array}$} & \multicolumn{2}{|c|}{$\begin{array}{l}\text { Degrés de liberté } \\
\text { (Degrees of freedom) }\end{array}$} & \multirow{2}{*}{$\begin{array}{l}\text { Estimations des carrés moyens } \\
\text { (Estimations of mean squares) }\end{array}$} & \multirow{2}{*}{$\begin{array}{l}\text { Test } F \\
(\mathrm{~F} \text { test) }\end{array}$} \\
\hline & Station (1) & Station (2) & & \\
\hline \multirow{4}{*}{ 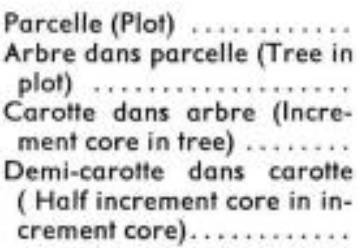 } & 149 & 147 & (1) & $(1) /(2)$ \\
\hline & 149 & 148 & $\sigma_{t}^{2}+k_{2} \sigma_{c}^{3}+k_{3} \sigma_{2}^{3}(2)$ & $(2) /(3)$ \\
\hline & 295 & 288 & $\sigma_{e}^{2}+k_{1} \sigma_{e}^{2}$ & $(3) /(4)$ \\
\hline & 590 & 567 & (4) & \\
\hline
\end{tabular}


avec

$k_{1}, k_{2}$ et $k_{3}$ dépendant du nombre d'observations et de leur ventilation suivant les sources de variation,

$\sigma_{\mathrm{e}}^{2}$ : variance « demi-carotte dans carotte $»$;

$\sigma_{\mathrm{e}}^{2}$ : variance « carotte dans arbre $"$;

$\sigma_{\mathrm{a}}^{2}$ : variance $\approx$ arbre dans parcelle $»$;

Le carré moyen au niveau «parcelle n n'est pas expiicité, étant donné que cette source de variation résulte d'un mélange composite de facteurs (provenance, répétition, bloc dans répétition).

Les covariances entre caractères se décomposent de la même façon ; on obtient facilement les corrélations entre demi-carottes dans la carotte, entre carottes dans l'arbre et entre arbres dans la parcelle.

- Dans le second cas, on avait un niveau de hiérarchie en moins (tabl. 6) :

TABLEAU 6

Décomposition de la variance pour l'infradensité Partitioning of variance for basic density

\begin{tabular}{|c|c|c|c|c|c|}
\hline \multirow{2}{*}{$\begin{array}{l}\text { Sources de variation } \\
\text { (Sources of variation) }\end{array}$} & \multicolumn{2}{|c|}{$\begin{array}{l}\text { Degrés de liberté } \\
\text { (Degrees of freedom) }\end{array}$} & \multirow{2}{*}{\multicolumn{2}{|c|}{$\begin{array}{l}\text { Estimations des carrés moyens } \\
\text { (Estimations of mean squares) }\end{array}$}} & \multirow{2}{*}{$\begin{array}{l}\text { Test } F \\
\text { ( } F \text { tests) }\end{array}$} \\
\hline & Station (1) & Station (2) & & & \\
\hline \multirow{3}{*}{ 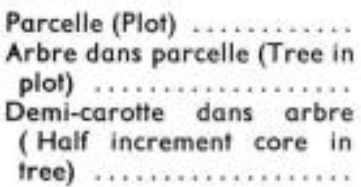 } & 149 & 147 & (1) & & (1)/(2) \\
\hline & 149 & 145 & $\sigma_{e}^{2}+k_{1} \sigma^{\mu}$ & (2) & $(2) /(3)$ \\
\hline & 289 & 257 & $\sigma_{\mathrm{e}}^{2}$ & (3) & \\
\hline
\end{tabular}

avec

$\sigma_{\mathrm{e}}^{2}$ : variance « demi-carotte dans arbre ».

$\sigma_{a}^{2}$; variance « arbre dans parcelle ».

Le tableau 7 donne, caractère par caractère et station par station, les résultats des analyses de variance (à l'exception des tests F pour l'effet parcelle : voir plus hout).

Pour le retrait axial, on remarque que la variabilité intra-arbre est significative dans les deux stations ; il en va différemment entre arbres, ce qui suggère l'intérêt d'une sélection individuelle pour ce caractère.

En revanche, pour les trois autres retraits, tout en étant très concordants, les résultats s'écartent sensiblement des précédents : Calabre,

- variabilité intra-arbre nulle statistiquement pour la Sicile, significative en

- variabilité individuelle toujours significative avec des valeurs de F plus importantes pour la station 1 que pour la station 2 (avec des degrés de liberté sensiblement égaux dans les deux cas). 
TABLEAU 7

Variabilité individuelle - résultałs des tests $F$ Individual variability - F tests results

\begin{tabular}{|c|c|c|c|}
\hline \multicolumn{2}{|c|}{$\begin{array}{c}\text { Caractères } \\
\text { (Characteristics) }\end{array}$} & \multirow{2}{*}{$\begin{array}{c}\text { Sources de variation } \\
\text { Entre carottes } \\
\text { dans l'arbre } \\
\text { (Increment core in tree) } \\
2,10 * * \\
1,61 * *\end{array}$} & \multirow{2}{*}{$\begin{array}{c}\text { (Sources of variation) } \\
\text { Entre arbres } \\
\text { dans la parcelle } \\
\text { (Tree in plot) } \\
1,22 \mathrm{NS} \\
1,53 *\end{array}$} \\
\hline $\begin{array}{l}\text { Retrait axial } \\
\text { (Axial shrinkage) }\end{array}$ & $\begin{array}{l}\text { Sicile } \ldots . . . \\
\text { Calabre .... }\end{array}$ & & \\
\hline $\begin{array}{l}\text { Retrait tangentiel } \\
\text { (Tangential shrinkage) }\end{array}$ & $\begin{array}{l}\text { Sicile ....... } \\
\text { Calabre ... }\end{array}$ & $\begin{array}{l}0,78 \mathrm{NS} \\
1,82 * \approx\end{array}$ & $\begin{array}{l}6,95 * * \\
3,41 * *\end{array}$ \\
\hline $\begin{array}{l}\text { Retrait radial } \\
\text { (Radial shrinkage) }\end{array}$ & $\begin{array}{l}\text { Sicile } \ldots . . . \\
\text { Calabre .... }\end{array}$ & $\begin{array}{l}1,11 \mathrm{NS} \\
1,84 * \bullet\end{array}$ & $\begin{array}{l}4,76 * * \\
3,35 * *\end{array}$ \\
\hline $\begin{array}{l}\text { Retrait volumétrique } \\
\text { (Volumetric shrinkage) }\end{array}$ & $\begin{array}{l}\text { Sicile ...... } \\
\text { Calabre .... }\end{array}$ & $\begin{array}{l}0,74 \mathrm{NS} \\
1,87 \cdots\end{array}$ & $\begin{array}{l}9,07 * * \\
4,00 * *\end{array}$ \\
\hline $\begin{array}{l}\text { Infradensité } \\
\text { (Basic density) }\end{array}$ & $\begin{array}{l}\text { Sicile } \ldots . . . \\
\text { Calabre .... }\end{array}$ & - & $\begin{array}{l}4,21 * * \\
2,68 * *\end{array}$ \\
\hline \multicolumn{4}{|c|}{ Degrés de liberté des F (Degrees of freedom) } \\
\hline & & Entre carottes dans l'arbre & $\begin{array}{l}\text { Entre arbres } \\
\text { dans la parcelle }\end{array}$ \\
\hline Retraits (Shrinkages) & $\begin{array}{l}\text { Sicile } \ldots . . . \\
\text { Calabre .... }\end{array}$ & $\begin{array}{l}295 \text { et } 590 \\
288 \text { et } 567\end{array}$ & $\begin{array}{l}149 \text { et } 295 \\
148 \text { et } 288\end{array}$ \\
\hline $\begin{array}{l}\text { Infradensité } \\
\text { (Basic density) }\end{array}$ & $\begin{array}{l}\text { Sicile ...... } \\
\text { Calabre .... }\end{array}$ & - & $\begin{array}{l}149 \text { et } 295 \\
145 \text { et } 257\end{array}$ \\
\hline
\end{tabular}

Au niveau arbre, les mêmes conclusions apparaissent pour l'infradensité.

Dans le tableau 8 nous avons fait figurer les moyennes des caractères, leurs coefficients de variation, les variances intra-carotte, entre carottes et entre arbres, ainsi que le coefficient de variation (rapport de l'écart type total à la moyenne en p. 100).

Nous sommes immédiatement frappés par les valeurs extrêmement fortes des retraits : 0,72 p. 100 pour le retrait axial, 28 p. 100 pour le retrait tangentiel, $7,84 p 100$ pour le retrait radial (moyennes des deux stations). A titre de comparaison, Nepveu, Keller et Teissier Du Cros (1978 op. cit.) ont observé sur vingt-huit clones de Peupliers euraméricains les valeurs suivantes : 0,05 p. 100 (retrait axial), 7,10 p. 100 (retrait tangentiel), 2,44 p. 100 (retrait radial) en précisant toutefois l'absence de collapse sur cette essence.

Les coefficients de variation sont globalement importants pour ces caractères, surtout pour le retrait axial, assez faibles pour l'infradensité avec une valeur moyenne de 7 à 8 p. 100 couramment observée pour le bois.

Pour les retraits, on observe que la variance intracarotte est très forte ; elle inclue certes les erreurs expérimentales, mais également des effets de l'orientement. Ces derniers sont donc plus importants que ceux de la position de l'échantillon le long d'une 
TABLEAU 8

Variabilité individuelle : décomposition de la variance en fonction des sources de variation

Individual variability : variance partitioning according to sources of variation

\begin{tabular}{|c|c|c|c|c|c|c|c|c|c|c|}
\hline \multirow{2}{*}{$\begin{array}{c}\begin{array}{c}\text { Caractères } \\
\text { (Characte- } \\
\text { ristics) }\end{array} \\
\text { Retrait axial. }\end{array}$} & \multirow{2}{*}{$\begin{array}{c}\begin{array}{c}\text { Stations } \\
\text { (Sta- } \\
\text { tions) }\end{array} \\
\\
\\
1 \\
2\end{array}$} & \multirow{2}{*}{$\begin{array}{c}\begin{array}{c}\text { Moyennes } \\
\text { générales } \\
\text { (General } \\
\text { means) }\end{array} \\
0,66 \text { p. } 100 \\
0,78 \text { p. } 100\end{array}$} & \multicolumn{2}{|c|}{$\begin{array}{c}\text { Variances } \\
\text { intra-carotte } \\
\text { (Within-increment } \\
\text { core variances) }\end{array}$} & \multicolumn{2}{|c|}{$\begin{array}{c}\text { Variance dans l'arbre } \\
\text { entre carottes } \\
\text { (Within-tree variances } \\
\text { between increment cores) }\end{array}$} & \multicolumn{2}{|c|}{$\begin{array}{c}\text { Variances entre arbres } \\
\text { dans la parcelle } \\
\text { (Within-plot variances } \\
\text { between trees) }\end{array}$} & \multirow{2}{*}{$\begin{array}{c}\text { Variances totales } \\
\text { (Total variances) } \\
\\
\\
0,2885 \\
0,8023\end{array}$} & \multirow{2}{*}{$\begin{array}{l}\text { Coefficients } \\
\text { de variation } \\
\text { (Coefficients } \\
\text { of variation) } \\
81 \text { p. } 100 \\
115 \text { p. } 100\end{array}$} \\
\hline & & & $\begin{array}{l}0,1785 \\
0,5238\end{array}$ & $\begin{array}{l}(62) \\
(65)\end{array}$ & $\begin{array}{l}0,0990 * * \\
0,1620 * *\end{array}$ & $\begin{array}{l}(34) \\
(20)\end{array}$ & $\begin{array}{l}0,0110 \text { NS } \\
0,1165^{*}\end{array}$ & $\begin{array}{r}(4) \\
(15)\end{array}$ & & \\
\hline $\begin{array}{c}\text { Retrait tan- } \\
\text { gentiel ..... }\end{array}$ & $\begin{array}{l}1 \\
2\end{array}$ & $\begin{array}{l}30,65 \text { p. } 100 \\
25,34 \text { p. } 100\end{array}$ & $\begin{array}{l}22,4795 \\
22,5392\end{array}$ & $\begin{array}{l}(46) \\
(39)\end{array}$ & $\begin{array}{l}0 \quad \text { NS } \\
9,3788^{* *}\end{array}$ & $\begin{array}{l}(0) \\
(16)\end{array}$ & $\begin{array}{l}26,3224 * * \\
25,6908 * *\end{array}$ & $\begin{array}{l}(54) \\
(45)\end{array}$ & $\begin{array}{l}48,8019 \\
57,6088\end{array}$ & $\begin{array}{l}23 \text { p. } 100 \\
30 \text { p. } 100\end{array}$ \\
\hline Retrait radial & $\begin{array}{l}1 \\
2\end{array}$ & $\begin{array}{l}8,57 \text { p. } 100 \\
7,11 \text { p. } 100\end{array}$ & $\begin{array}{l}2,1160 \\
2,1894\end{array}$ & $\begin{array}{l}(47) \\
(39)\end{array}$ & $\begin{array}{l}0,1216 \text { NS } \\
0,9362 \ldots\end{array}$ & $\begin{array}{l}(3) \\
(17)\end{array}$ & $\begin{array}{l}2,2437 * * \\
2,4655 * *\end{array}$ & $\begin{array}{l}(50) \\
(44)\end{array}$ & $\begin{array}{l}4,4813 \\
5,5911\end{array}$ & $\begin{array}{l}25 \text { p. } 100 \\
33 \text { p. } 100\end{array}$ \\
\hline $\begin{array}{c}\text { Retrait volu- } \\
\text { métrique ... }\end{array}$ & $\begin{array}{l}1 \\
2\end{array}$ & $\begin{array}{l}43,03 \text { p. } 100 \\
35,50 \text { p. } 100\end{array}$ & $\begin{array}{l}35,4791 \\
30,3939\end{array}$ & $\begin{array}{l}(40) \\
(35)\end{array}$ & ${ }_{13}^{0} \quad$ NS & $\begin{array}{l}(0) \\
(15)\end{array}$ & $\begin{array}{l}53,4995 * * \\
44,3794 * *\end{array}$ & $\begin{array}{l}(60) \\
(50)\end{array}$ & $\begin{array}{l}88,9786 \\
88,1951\end{array}$ & $\begin{array}{l}22 \text { p. } 100 \\
26 \text { p. } 100\end{array}$ \\
\hline Densité....... & $\begin{array}{l}1 \\
2\end{array}$ & $\begin{array}{l}503 \mathrm{~g} / \mathrm{dm}^{3} \\
516 \mathrm{~g} / \mathrm{dm}^{8}\end{array}$ & & & $\begin{array}{l}466,21 \\
787,86\end{array}$ & $\begin{array}{l}(38) \\
(52)\end{array}$ & $\begin{array}{l}765,33 * * \\
716,44 * *\end{array}$ & $\begin{array}{l}(62) \\
(48)\end{array}$ & $\begin{array}{l}1231,54 \\
1504,30\end{array}$ & $\begin{array}{l}7 \text { p. } 100 \\
8 \text { p. } 100\end{array}$ \\
\hline
\end{tabular}

- Station 1 - Sicile; Station 2 - Calabre.

- Entre parenthèses figure le pourcentage de la variabilite tolale expliqué par l'effet controlé, (Figures between brackets give the percent of total variability explained by the effect studied.

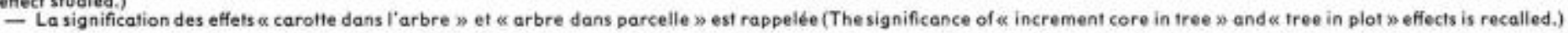


génératrice de l'arbre (appréciés par la variabilité entre carofte dans l'arbre). Pour le retrait axial, notons entre Sicile et Calabre une grande différence entre les variances intra $(0,1785$ contre 0,5258$)$; ceci peut s'expliquer par le fait qu'en Sicile, les arbres sont sur terrain plat alors qu'en Calabre, un grand nombre d'entre eux, plantés sur une pente présentent un bois anisotrope pour le caractère considéré alors que le phénomène n'est pas sensible dans les directions tangentielles et radiales (en Calabre, la direction du prélèvement des carottes était, en gros, parallèle à la ligne de plus grande pente).

Un phénomène analogue à celui observé pour le retrait axial peut sans doute rendre compte du fait que, pour l'infradensité, les variances entre carottes sont égales respectivement à 466 et 788 en Sicile et en Calabre ; en effet, on a vu que pour ce critère, la manière de procéder à l'échantillonnage conduit à inclure dans la variabilité entre carottes (ou plutôt ici demi-carotte) l'effet de l'orientement, d'où la valeur notablement plus forte notée en Calabre.

\subsection{Efude des corrélations.}

Les tableaux 9,10 et 11 décomposent les corrélations aux niveaux « demi-carotte dans carotte », "carotte dans arbre » et " arbre ». Entre parenthèses figurent celles dépourvues de signification (test $F$ non significatif pour l'un ou l'autre des deux caractères pour la source de variation considérée).

\section{TABLEAU 9}

Corrélations enfre caractères au niveau demi-carofte dans carotte

Correlations befween characteristics ( $\alpha$ half-increment core in increment core $x$ )

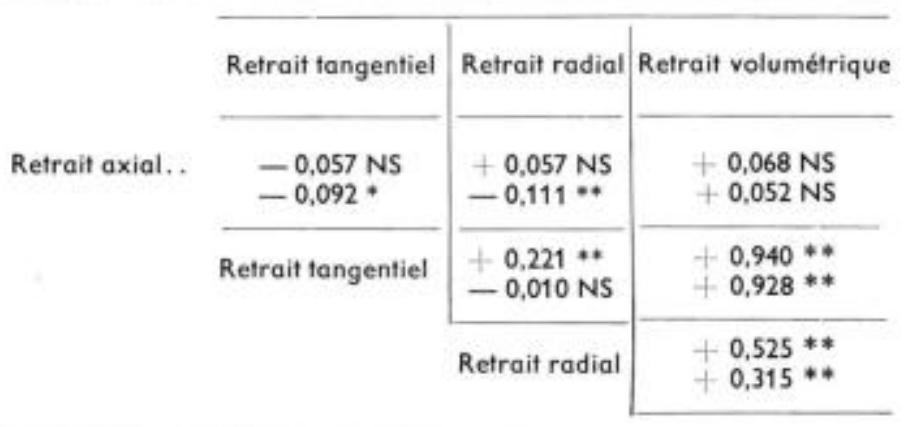

1) Chiffres du haut (First numbers) : station 1 (Sicile).

Chiffres du bas (Second numbers) ; station 2 (Calabre).

2) Sevils de signification : le nombre de degrés de liberté pour r esł pris égal à celui du carré moyen au niveau \&s sous échantillon dans l'échantillon os (Significance limits : the number of degrees of freedom for $r$ is that of mean square $\alpha$ half increment core in increment core o.)

\begin{tabular}{lcc}
\hline & Sicile & Calabre \\
\hline $\begin{array}{l}\text { Corrélations entre re- } \\
\text { traits ............. }\end{array}$ & 590 & $\$ 67$ \\
\hline
\end{tabular}


3.221. Corrélations au niveau demi-carotte dans carotte (tabl. 9).

Elles sont fortes entre retrait tangentiel et retrait volumétrique d'une part, retrait radial et retrait volumétrique d'autre part, ce qui ne nous étonne guère puisque le second terme est calculé à partir des deux premiers (voir chapitre « Matériel et Méthode $s)$. Le retrait axial qui reste faible intervient peu dans les variations du retrait volumétrique. A noter une corrélation positive en Sicile entre retrait tangentiel et retrait radial $\left(+0,221^{* *}\right)$ qui $n^{\prime}$ 'apparait plus en Calabre. Les autres valeurs sont nulles ou très proches des seuils de signification.

\subsection{Corrélations au niveau carotte dans arbre (łabl. 10).}

L'absence d'effet "échantillon dans l'arbre » sur de nombreux caractères en Sicile nous interdit d'accorder un quelconque crédit pour cette station aux liaisons calculées. En Calabre, on note une liaison positive retrait tangentiel-retrait axial ; aucune valeur significative à 1 p. 100 n'apparaît entre les retraits ef l'infradensité.

TABLEAU 10

Corrélations entre caractères au niveau * carolle dans arbre $⿻$ (demi-carotte dans arbre pour corrélations refraits-infradensité)

Correlations between characteristics ( $\alpha$ increment core in tree $x)$ (half-increment core in tree for correlations between shrinkages and basic density)

\begin{tabular}{|c|c|c|c|c|}
\hline & & & & \\
\hline \multirow{5}{*}{ Retrait axial } & \multirow{2}{*}{$\begin{array}{c}\text { Retrait tangentiel } \\
\begin{array}{c}(0) \\
+0.400 * *\end{array}\end{array}$} & \multirow{2}{*}{$\begin{array}{l}\text { Retrait radial } \\
\begin{array}{l}(-0,434) \\
+0,038 \mathrm{NS}\end{array}\end{array}$} & \multirow{2}{*}{$\begin{array}{c}\text { Refrait volumétrique } \\
\begin{array}{c}(0) \\
+0,506 *\end{array}\end{array}$} & \multirow{2}{*}{$\begin{array}{l}\text { Infradensité } \\
\begin{array}{l}0,234 * * \\
+0,151 *\end{array}\end{array}$} \\
\hline & & & & \\
\hline & \multirow[t]{3}{*}{ Retrait tangentiel } & $\begin{array}{c}(0) \\
-0,034 \mathrm{NS}\end{array}$ & $\begin{array}{c}(0) \\
+0.934 * *\end{array}$ & $\begin{array}{l}(-0,112) \\
+0,008 \mathrm{NS}\end{array}$ \\
\hline & & \multirow[t]{2}{*}{ Retrait radial } & $\begin{array}{c}(0) \\
+0,302 \ldots\end{array}$ & $\begin{array}{l}(+0,033) \\
+0,043 \mathrm{NS}\end{array}$ \\
\hline & & & Retrait volumétrique & $\begin{array}{l}(-0,066) \\
+0,043 \mathrm{NS}\end{array}$ \\
\hline
\end{tabular}

1) Chiffres du haut (First numbers) : station 1 (Sicile).

Chiffres du bas (Second numbers) : station 2 (Calabre).

2) Seuils de signification : le nombre de degrés de liberté pour $r$ est pris égal à celui du carré moyen au niveau « carotte dans l'arbre 3 (Significance limits : the number of degrees of freedom for $r$ is that of mean square $\alpha$ increment core in tree $x$.)

\begin{tabular}{lcc}
\hline \hline & Sicile & Calabre \\
\hline Corrélations entre retraits & 295 & 288 \\
$\begin{array}{l}\text { Corrélations entre infra- } \\
\text { densité et retraits ........ }\end{array}$ & 289 & 257 \\
\hline
\end{tabular}

3) Entre parenthèses : corrélations dépourvues desens (effet $\alpha$ échantillon dans l'arbre $\%$ non significatif) (Between brackets : correlations without meaning ( $\alpha$ tree in plot $x$ effect without significance)). 


\subsection{Corrélations au niveau de l'arbre (tabl. 11).}

Elles sont les plus intéressantes sur le plan pratique. Hormis le cas du retrait axial, les valeurs observées en Sicile et en Calabre sont très proches statistiquement. D'une façon générale, on observe des liaisons positives entre les différents retraits et négatives entre ces derniers et l'infradensité. Ceci peut s'expliquer par le fait que les retraits considérés sont des retraits dus au collapse. On sait que ce phénomène se traduit par un effondrement des parois cellulaires qui est moins prononcé quand ces dernières sont épaisses, donc le bois plus dense (Kauman, 1966).

\section{TABLEAU 11}

Corrélations entre caractères au niveau de l'arbre

Correlations between characteristics ( $\alpha$ tree in plot $\mathrm{x}$ )

1) Chiffres du haut (First numbers) : station 1 (Sicile).

Chiffes du bas (Second numbers) : station 2 (Calabre).

2) Seuils de signification : le nombre de degrés de liberté pour r est pris égal a celui du carré moyen au niveau « arbre dans la parcelle $\%$. (Significance limits t the number of degrees of freedom for $r$ is that of mean square $\alpha$ tree in plot 3 ).

\begin{tabular}{lcc}
\hline & Sicile & Calabre \\
\hline Corrélations entre retraits & 149 & 148 \\
$\begin{array}{l}\text { Corrélations entre infra- } \\
\text { densité et retraits....... }\end{array}$ & 149 & 145 \\
\hline
\end{tabular}

3) Entre parenthèses : corrélations dépourvues de sens (effet arbre non significatif) (Befween brackets : correlations without meaning ( $\alpha$ tree in plot $x$ effect without significance)).

\section{3. - Etude de la variabilité infraspécifique}

\subsection{Analyse de variance.}

Pour les deux stations, on a étudié les effets «provenance» (24 degrés de liberté), « répétition » (5 degrés de liberté), « bloc la répétition » (24 degrés de liberté). Du fait de l'absence de certains individus pour diverses raisons, l'erreur a, pour les 
retraits, 1130 degrés de liberté en Sicile, 1097 en Calabre, et pour l'infradensité, respectivement 534 et 496 degrés de liberté. Ces nombres très voisins autorisent donc le rapprochement des $\mathrm{F}$ calculés pour chaque dispositif. Les résultats des analyses de variance figurent dans le tableau 12 .

\section{TABLEAU 12}

Effets provenance, répétition et bloc. Résultats des analyses de variance. Valeurs des $\mathrm{F}$ calculés et signification Provenance, replication and block effects. Results of variance analysis. F values and significance

\begin{tabular}{|c|c|c|c|c|}
\hline & & \multicolumn{3}{|c|}{$\begin{array}{l}\text { Sources de variation } \\
\text { (Sources of variation) }\end{array}$} \\
\hline \multicolumn{2}{|c|}{ Caractères } & $\begin{array}{l}\text { Provenance } \\
\text { (Provenance) }\end{array}$ & $\begin{array}{l}\text { Répétition } \\
\text { (Replication) }\end{array}$ & $\begin{array}{c}\text { Bloc dans répétition } \\
\text { (Bloc } \\
\text { in replication) }\end{array}$ \\
\hline Retrait axial & $\begin{array}{l}\text { Sicile ............. } \\
\text { Calabre ....... }\end{array}$ & $\begin{array}{l}7,88 * * \\
6,49 \cdots\end{array}$ & $\begin{array}{l}5,59 * * \\
5,79 * *\end{array}$ & $\begin{array}{l}1,97 * \\
2,61 *\end{array}$ \\
\hline Retrait tangentiel & $\begin{array}{l}\text { Sicile ............. } \\
\text { Calabre ...... }\end{array}$ & $\begin{array}{l}81,24 * * \\
47,33 \cdots\end{array}$ & $\begin{array}{r}2,89 * * \\
28,16 * *\end{array}$ & $\begin{array}{l}4,14 * * \\
3,52 * *\end{array}$ \\
\hline Retrait radial & $\begin{array}{l}\text { Sicile ............ } \\
\text { Calabre ...... }\end{array}$ & $\begin{array}{l}29,11 * * \\
15,81 * *\end{array}$ & $\begin{array}{l}11,23 * * \\
35,16 * *\end{array}$ & $3,17 * *$ \\
\hline Retrait volumétrique & $\begin{array}{l}\text { Sicile ............. } \\
\text { Calabre ....... }\end{array}$ & $\begin{array}{l}81,10 * * \\
51,63 * *\end{array}$ & $\begin{array}{r}5,57 * * \\
39,77 * *\end{array}$ & $4,25 * *$ \\
\hline Infradensités & $\begin{array}{l}\text { Sicile ............ } \\
\text { Calabre ....... }\end{array}$ & $21,80 \cdots$ & $\begin{array}{l}0,38 \text { NS } \\
4,83^{* *}\end{array}$ & $\begin{array}{l}1,63^{*} \\
1,99^{* *}\end{array}$ \\
\hline
\end{tabular}

Pour le retrait axial, on observe des résultats très voisins pour les stations 1 et 2 avec des effets très significatifs pour les trois sources de variation.

En revanche, pour les trois autres retraits et l'infradensité, hormis le cas du facteur « bloc dans répétition », les F sont très différents en Sicile et en Calabre. Pour les trois premiers, il semble que les effets « provenance » soient moins accusés en Calabre. Pour le cas de la répétition, c'est l'inverse. Pour l'infradensité, si les $F$ sont très voisins au niveau « provenance ", l'effet répétition n'est significatif qu'en Calabre.

Ceci n'est pas étranger au fait que le dispositif est beaucoup plus hérétogène en Calabre, ce qui, en plus d'augmenter les effets « répétition », doit masquer ceux des «provenances» par le biais d'interactions génotype-environnement.

Dans l'ordre, les $F$ « provenance » sont les plus forts pour le retrait tangentiel et volumétrique $(47,33$ à 81,24$)$ suivis de l'infradensité et du retrait radial $(15,81$ à $29,11)$. Les valeurs sont plus faibles pour le retrait axial $(6,49$ à 7,88$)$.

Le tableau 13 présente les moyennes de provenances pour les deux stations. $Y$ figurent également les classements (chiffres en italiques) ainsi que les différences significatives à 5 p. 100 calculées à l'aide du test de Tukey, le dispositif étant peu déséquilibré. Nous signalons également des circonférences ef hauteurs prises en septembre 1976. Malheureusement les tests n'ont pas été effectués sur ces variables car les données disponibles n'étaient que des moyennes de provenances. 
Moyennes et classements des provenances pour les deux stations

Means and ranks of provenances for the two sites

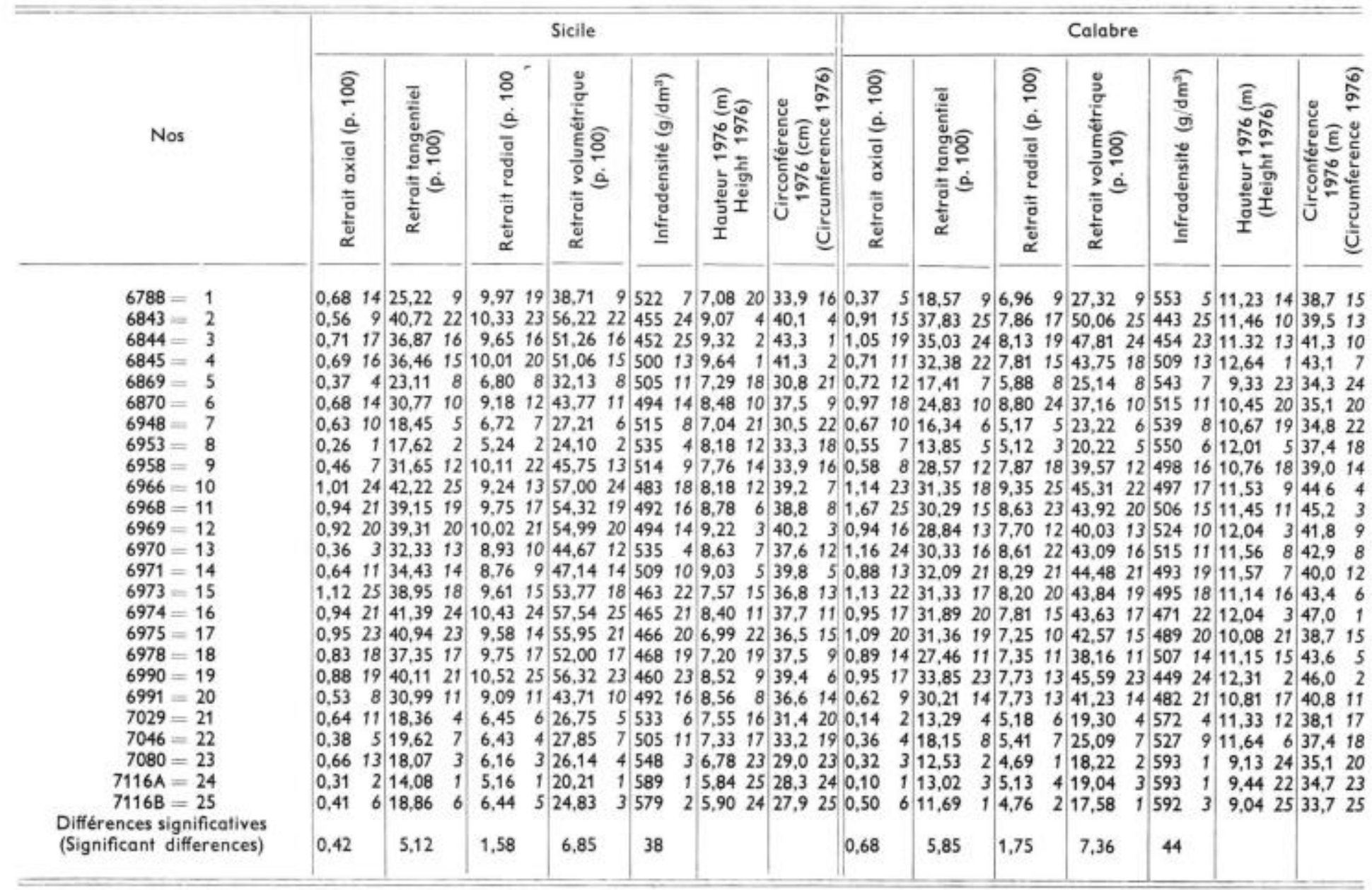


On est aussitôt frappé par l'énorme variabilité entre origines, ce que les analyses de variance nous laissaient supposer. Ainsi les retraits volumétriques peuvent varier de 20,21 p. 100 à 57,54 p. 100 en Sicile, de 17,58 p. 100 à 50,06 p. 100 en Calabre. Pour l'infradensité, les valeurs extrêmes sont 452 et $589 \mathrm{~g} / \mathrm{dm}^{3}$ (station 1), 443 et $593 \mathrm{~g} / \mathrm{dm}^{3}$ (station 2).

Ceci illustre l'intérêt évident d'une sélection infraspécifique : en particulier, il est clair, si l'on considère le retrait volumétrique, qu'une valeur de 58 p. 100 interdit tout usage noble (à moins d'un reconditionnement) alors que 18 p. 100 (avec collapse) correspond à une essence française d'assez bon comportement au séchage telle que le hêfre (Bordeaux, 1977 ; Allegret, 1976), d'oủ les conséquences économiques que l'on imagine.

On remarque que la provenance 4 (6845, Lac Albacutya), signalée par Lacaze (1970 op. cit.) pour sa vigueur remarquable, présente un refrait fort et une infradensité assez faible.

\subsection{Corrélations au niveau des moyennes de provenances.}

On les trouve dans le tableau 14. Elles sont pratiquement toutes significatives, très proches d'une station à l'autre, et confirment celles observées au niveau individuel dans le tableau 11. D'une façon générale, on note des valeurs positives entre les retraits, entre les retraits ef la vigueur, négatives entre les retraits et l'infradensité, entre la vigueur et l'infradensité.

\section{TABLEAU 14}

Corrélations entre moyennes de provenances

Correlations between means of provenances

\begin{tabular}{|c|c|c|c|c|c|c|}
\hline & $\begin{array}{c}\text { Retrait } \\
\text { tangentiel }\end{array}$ & $\begin{array}{l}\text { Retrait } \\
\text { radial }\end{array}$ & $\begin{array}{c}\text { Retrait } \\
\text { voluméfrique }\end{array}$ & Infradensité & $\begin{array}{l}\text { Hauteur } \\
1976\end{array}$ & $\begin{array}{c}\text { Circonférence } \\
1976\end{array}$ \\
\hline \multirow[t]{6}{*}{ Retrait axial } & $\begin{array}{l}+0,738 * * \\
+0,742 * *\end{array}$ & $\begin{array}{r}0,653 * \\
+0,779 *\end{array}$ & $\begin{array}{r}+0,745 * \\
+0,778 *\end{array}$ & $\begin{array}{l}-0,636 * * \\
-0,678 * *\end{array}$ & $\begin{array}{r}+0,270 \mathrm{NS} \\
+0,351 \mathrm{NS}\end{array}$ & $\begin{array}{l}+0,532 * * \\
+0,643 * *\end{array}$ \\
\hline & $\begin{array}{c}\text { Retrait } \\
\text { tangentiel }\end{array}$ & $\begin{array}{r}0,910 * * \\
+\quad 0,883 * *\end{array}$ & $\begin{array}{r}+0,997 * \\
+0,995 *\end{array}$ & $\begin{array}{l}-0,839^{* *} \\
-0,932^{* *}\end{array}$ & $\begin{array}{r}+0,657 \cdots \\
+0,566 \cdots\end{array}$ & $\begin{array}{l}+0,878 * * \\
+0,762 * *\end{array}$ \\
\hline & & $\begin{array}{l}\text { Retrait } \\
\text { radial }\end{array}$ & $\begin{array}{l}+0,936 * \\
+0,919 * *\end{array}$ & $\begin{array}{l}-0,764^{* *} \\
-0,718^{* *}\end{array}$ & $\begin{array}{r}+0,616 \cdots \\
+0,505 \cdots\end{array}$ & $\begin{array}{l}+0,812 * * \\
+0,715 * *\end{array}$ \\
\hline & & & $\begin{array}{c}\text { Retrait } \\
\text { volumétrique }\end{array}$ & $\begin{array}{l}-0,840 * * \\
-0,920 * *\end{array}$ & $\begin{array}{r}0,652 * * \\
+0,559 * \cdots\end{array}$ & $\begin{array}{l}+0,874 * * \\
+0.768 * *\end{array}$ \\
\hline & & & & Infradensité & $\begin{array}{l}-0,585^{* *} \\
-0,576^{* *}\end{array}$ & $\begin{array}{l}-0,778 * \\
-0,683 *\end{array}$ \\
\hline & & & & & $\begin{array}{c}\text { Hauteur } \\
1976\end{array}$ & $\begin{array}{l}+0,879 \cdots \\
+0,742 * *\end{array}$ \\
\hline
\end{tabular}

1) Premiers chiffres (First numbers) : station 1 (Sicile).

Deuxièmes chiffres (Second numbers) : station 2 (Calabre).

2) Seuils de signification du coefficient de corrélation (24 degrés de liberté) (Significance limits of coefficient of correlation ( 24 degrees of freedom)).

$0,388(5$ p. 100).

0.496 (1 p. 100) 


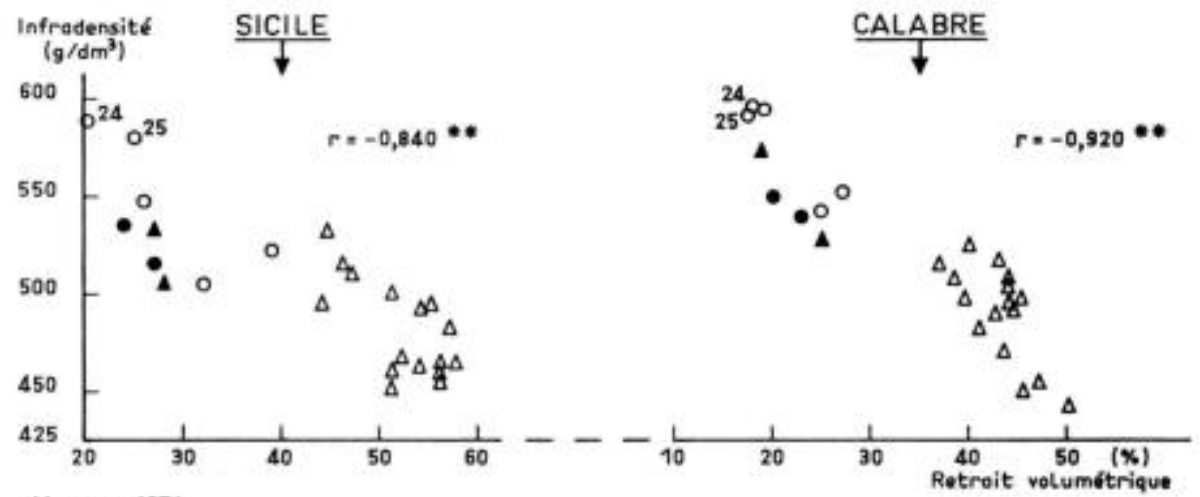

Hauteur 1976

(m)

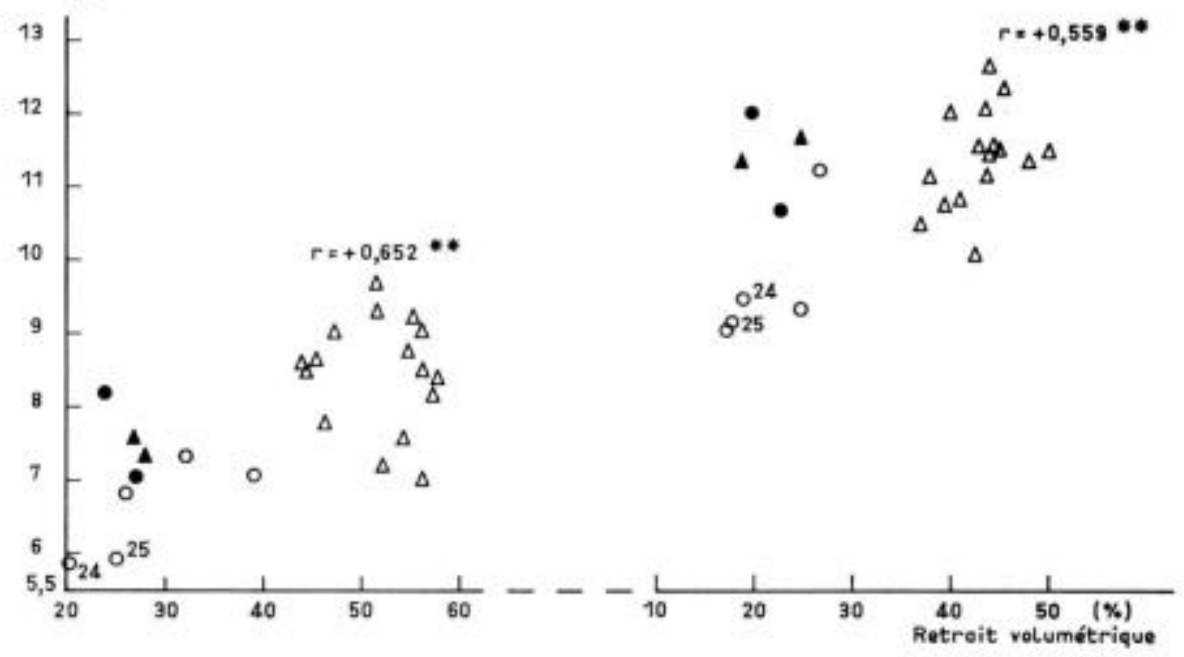

Infradensité

$\left(\mathrm{g} / \mathrm{dm}^{3}\right)$
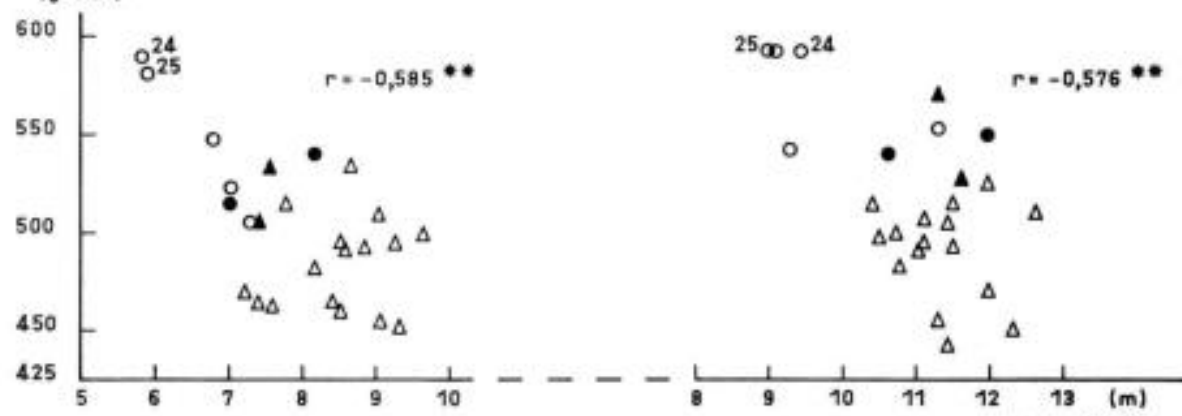

- Northern Territory

- Northern Queenstend

$\Delta \Delta_{\Delta \Delta \Delta}^{\Delta \Delta} \Delta$ ${ }_{\Delta}^{\Delta \Delta} \frac{\Delta \Delta}{\Delta} \Delta$ $\Delta \Delta \Delta$

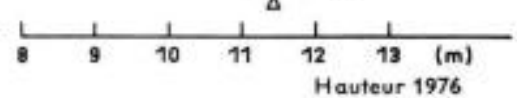

FIG. 2. - Corrélations entre moyennes de provenances.

Correlations between means of provenances.

Infradensité (basic density) ; Retrait volumétrique (volumetric shrinkage) ; Hauteur (height). 
II semble donc qu'une sélection exclusivement dirigée vers la production en volume fera chuter l'infradensité et croître dangereusement les retraits au point de risquer de cantonner cette essence dans les usages peu nobles. Rappelons toutefois que nous parlons ici de retraits avec collapse ; il n'est pas impossible qu'un reconditionnement bien étudié puisse lever ce grave défaut.

La figure 2 illustre pour les deux stations quelques corrélations entre :

- infradensité et retrait volumétrique :

- hauteur 1976 et retrait volumétrique :

- infradensité et hauteur 1976.

On a pris soin dans ce cas de signaler par quatre symboles différents les grandes régions de provenances concernées. On note une remarquable ségrégation des origines du Murray River System, les trois autres groupes étant assez voisins les uns des autres.

On peut aussi observer que les provenances 24 (7116A) et 25 (7116B), qui sont en réalité une seule et même origine, sont très proches (ces deux origines avaient été artificiellement créées à partir d'un même lot de graines afin de satisfaire aux contraintes du dispositif).

D'une façon générale, on peut dire que cette grande région du bassin de la rivière Murray et Australie Méridionale à climat à tendance méditerranéenne se caractérise pour $E$. camaldulensis par une infradensité faible, une croissance ef un retrait volumétrique élevés.

Les provenances jugées bonnes pour la qualité du bois appartiennent à des groupes d'Eucalyptus camaldulensis très différents de ceux de la zone généralement prospectée (Murray River). II s'agit, notons-le, de provenances tropicales finalement peu adaptées aux conditions méditerranéennes, en particulier pour la résistance au froid (7116) ou d'une provenance, la 6953, récoltée probablement par erreur et qui appartient en fait à l'espèce tereticornis ou à une espèce très voisine (Lacaze, communication personnelle).

\section{4. - Etude de l'effet «station » (tabl. 15)}

\subsection{Comparaison des moyennes.}

On a comparé les moyennes de provenances en Sicile et en Calabre par la méthode des couples. L'effet station est significatif sur tous les caractères, à l'exception du retrait axial. Dans la station 1, les autres retraits sont supérieurs à ceux de la station 2 ; la vigueur ef l'infradensité sont inférieures. A ce niveau, ces résultats témoignent d'une liaison négative retraits-infradensité analogue aux résultats des tableaux 11 et 14 . II en va tout autrement pour les corrélations retrait-vigueur et infradensitévigueur : en passant de Sicile en Calabre, le retrait diminue, l'infradensité augmente, la vigueur également.

Ceci témoigne pour ces deux couples de caractères de liaisons génétiques (effet provenance) et environnementales (effet station) de signes opposés, d'où l'intérêt pour le sélectionneur forestier de bien séparer ces deux sources de variation. On commettrait par exemple une grossière erreur en déduisant de la valeur interprovenance positive entre vigueur et retrait qu'une amélioration du milieu (par le choix d'une 


\section{TABLEAU 15}

Effets de la station sur différents caractères (comparaisons de moyennes par la méthode des couples) Effects of sile an various characteristics (comparison of means with fest of differences)

\begin{tabular}{|c|c|c|c|}
\hline Caractères & $\begin{array}{l}\text { Valeurs du t calculé } \\
\text { et signification } \\
\text { (t values and significance) }\end{array}$ & $\begin{array}{l}\text { Moyennes Sicile } \\
\text { (Sicily means) }\end{array}$ & $\begin{array}{c}\text { Moyennes Calabre } \\
\text { (Calabria means) }\end{array}$ \\
\hline 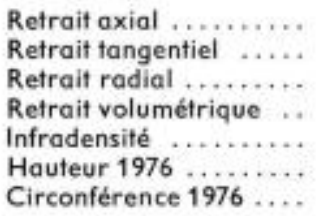 & $\begin{array}{r}1,93 \text { NS } \\
8,38 * * \\
7,97 * * \\
8,99 * * \\
3,45 * * \\
22,10 * * \\
6,98 * *\end{array}$ & $\begin{array}{c}0,66 \text { p. } 100 \\
30,60 \text { p. } 100 \\
8,57 \text { p. } 100 \\
42,94 \text { p. } 100 \\
503 \mathrm{~g} / \mathrm{dm}^{3} \\
7,93 \mathrm{~m} \\
35,78 \mathrm{~cm}\end{array}$ & $\begin{array}{r}0,77 \text { p. } 100 \\
25,30 \text { p. } 100 \\
7,10 \text { p. } 100 \\
35,41 \text { p. } 100 \\
516 \mathrm{~g} / \mathrm{dm}^{3} \\
11,05 \mathrm{~m}^{3} \\
39,85 \mathrm{~cm}\end{array}$ \\
\hline
\end{tabular}

Valeur du $t$ calculé à 24 degrés de liberté ( 1 value with 24 degrees of freedom) :

2,10 (5 p. 100).

$2,80$ (1 p. 100$)$.

station, d'une sylviculture ou d'une fertilisation) conduirait ipso facto à une détérioration de la qualité du bois : c'est en effet le contraire qui se produit.

Ce genre de phénomène -opposition entre effets du milieu et des gènes-a d'ailleurs été observé par l'un de nous (Nepveu, 1973 op. cit.) à propos des corrélations hauteurdensité du bois sur pin maritime ; on observe un coefficient positif au niveau génétique, négatif au niveau du milieu. II serait particulièrement intéressant de reprendre ce genre de calculs à l'intérieur des provenances en étudiant par exemple un test de descendances ou de clones qui nous permettrait de séparer les covariances génétiques (ou génotypiques) et environnementales.

En effet, il n'est pas certain qu'un supplément de vigueur apporté par la sélection de génotypes individuels ait également des conséquences défavorables sur la qualité du bois; on ne peut absolument pas confondre les corrélations génétiques aux niveaux population et individuel.

Remarque : Au chapitre «Matériel et Méthode», on a souligné que les échantillons provenant de Sicile avaient séjourné dans l'eau plus longtemps que ceux de Calabre. Ceci n'explique-t-il pas les différences entre les deux stations, notamment en ce qui concerne le retrait? A notre connaissance, aucune preuve expérimentale ne peut venir appuyer cette hypothèse ; en revanche, nous savons qu'un des slogans publicitaires destiné à vanter les mérites des fameux bois du Nord était leur faible retrait dô, d'après les importateurs, au fait qu'ils avaient été flottés, ce qui, parait-il, diminuait le « nerf du bois ». En nous basant sur cette opinion, à vrai dire peu scientifique, on peut conclure que ce n'est pas l'immersion prolongée des éprouvettes de Sicile, bien au contraire, qui peut expliquer les différences entre stations.

\subsection{Interactions génotype-environnement.}

On se reportera d̀ la figure 3 .

II est intéressant de constater l'existence d'une forte variabilité infraspécifique. II faut, pour l'exploiter, que le classement des provenances reste à peu près constant 


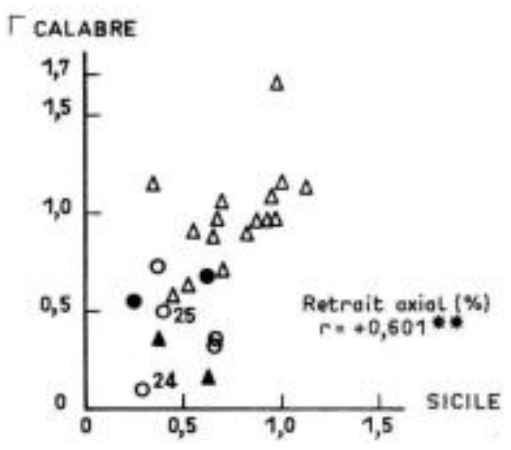

\section{CALABRE}
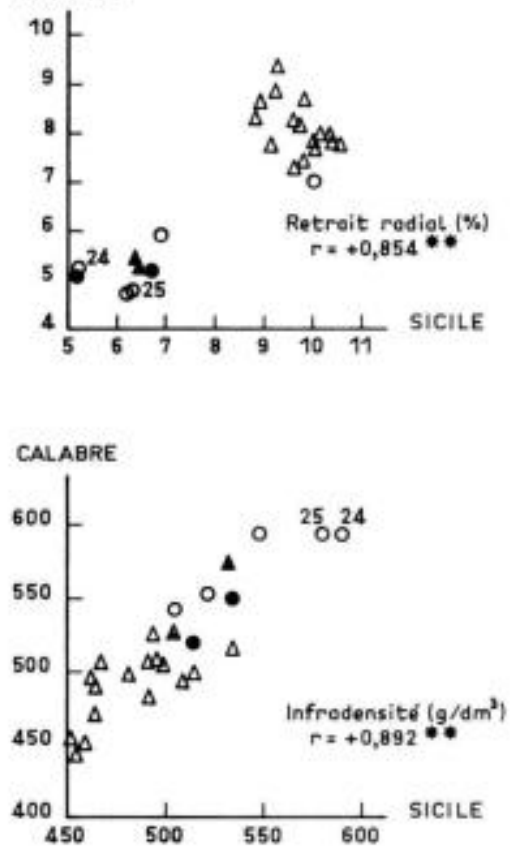

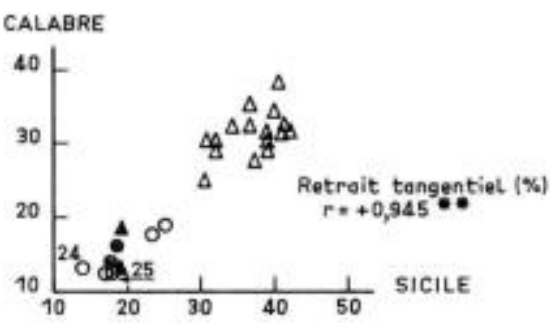

CALABRE

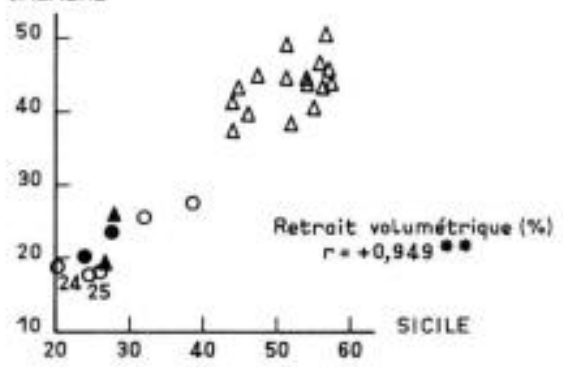

CALABRE

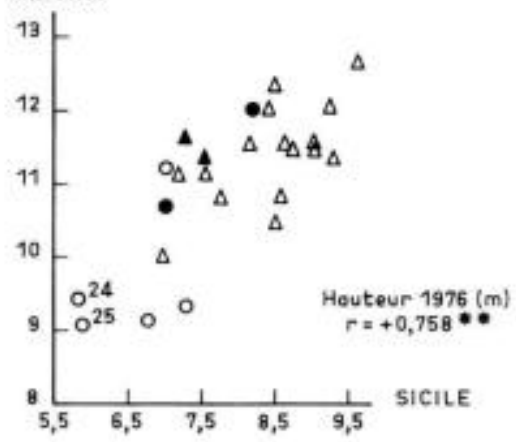

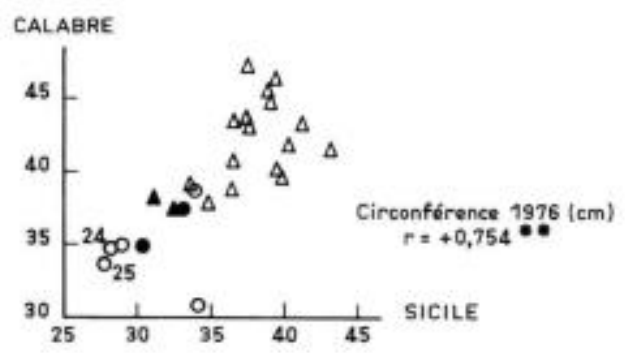

- Northern Territory

- Northern Queensland
$\Delta$ Murray River System

A Western Austrelia

FIG. 3. - Interactions gênotype-environnement.

Genotype environment interactions.

Retrait axial (axial shrinkage) ; Retrait radial (radial shrinkage) ;

Retrait volumétrique (volumetric shrinkage) ; Retrait tangentiel (tangential shrinkage) : Hauteur (height) ; Infradensité (basic density) ; Circonférence (circumference). 
suivant les stations, c'est-à-dire que les interactions génotype-environnement soient faibles. Sinon, on se voit contraint, soit de choisir des provenances remarquables par leur stabilité, soit de délimiter des milieux écologiques à l'intérieur desquels les résultats de chaque dispositif expérimental sont extrapolables. Dans le premier cas, le gain génétique est naturellement affaibli ; dans le second, il faut procéder à des études de milieux souvent longues, difficiles et coûteuses en moyens matériels.

Afin de nous faire une opinion sur ces phénomènes interactifs, nous avons calculé les corrélations entre moyennes de provenances, en Sicile et en Calabre. Elles sont toutes significatives, très fortes pour les retraits tangentiel, radial, volumétrique et l'infradensité $(0,854$ à 0,949$)$, moins importantes pour la vigueur et le retrait axial.

La considération des graphiques doit cependant nous mettre en garde ; il faut en effet conserver à l'esprit que ces calculs sont relatifs à la totalité des vingt-cinq provenances considérées et éviter de généraliser à un sous-ensemble d'origines. Ainsi, pour le retrait radial, une bonne liaison apparait $(0,854)$; cependant, en prenant uniquement les provenances de Murray River System, on obtient un coefficient négatif $(-0,490)$ très proche du seuil de signification à 5 p. $100(-0,497)$.

\section{4. - Conclusion générale}

Ce travail, entrepris sur deux dispositifs italiens de l'essai international de comparaison de provenances d'Eucalyptus camaldulensis, a apporté des informations intéressantes qui confirment, sur des caractéristiques physiques importantes, retraits avec collapse et infradensité, la grande variabilité infraspécifique déjà observée pour la vigueur.

Nous avons mis en évidence dans un ensemble de provenances représentant l'aire très vaste de l'essence le caractère original d'une grande région : le bassin de la rivière Murray et l'Australie méridionale qui se distingue nettement des autres par des infradensités faibles, une croissance et un retrait élevés.

Les corrélations intercaractères au niveau provenance et au niveau individuel ont montré l'existence de liaisons négatives vigueur-infradensité et infradensitéretrait, positives entre vigueur et retrait. Ceci éclaire sans aucun doute d'un jour nouveau la stratégie d'amélioration de $E$. camaldulensis, basée jusqu'ici sur la vigueur, si l'on considère que le retrait volumétrique peut varier de 18 à 58 p. 100 barrant ainsi la route à l'utilisation noble de certaines origines. Notons cependant qu'en passant d'une station à une autre, on a pu, tout en augmentant l'infradensité et la vigueur, diminuer le retrait. II serait sans doufe intéressant de reprendre ce travail sur un nombre plus élevé de stations afin d'estimer les corrélations intercaractères à ce niveau.

Ces résultats pouvant incliner à certains égards l'améliorateur forestier au pessimisme, ne peuvent faire oublier cependant des données intéressantes, à savoir la faiblesse des interactions génotype-environnement et la grande variabilité individuelle qui peut justifier une sélection à l'intérieur des provenances, à condition naturellement qu'elle soit d'origine génétique.

Notons que le caractère calcifuge du camaldulensis est remis en question par 
ses bons résultats en Calabre au point de vue croissance en hauteur et en circonférence et densité du bois dont l'ensemble conditionne la productivité nette en tonnes de matières sèches par hectare et par an.

Reçu pour publication en février 1978.

\section{Remerciements}

Nous remercions très vivement MM. Lacaze, Geminiani et Scaramuzzi, ainsi que les techniciens de l'Ente Nazionale per la Cellulosa e per la Carta pour l'aide qu'ils nous ont apportée dans la récolte des échantillons. Notre gratitude va également à M. Xeuxet de la Station de Biométrie du C.N.R.F. pour le traitement des données.

\section{Summary}

Infraspecific variability of shrinkage with collapse and wood density in Eucalyptus camaldulensis

Twenty four provenances of Eucalyptus camaldulensis from two provenance tests located in ltaly (Sicily and Calabria) were investigated for shrinkage with collapse and for wood density. These characteristics were measured on a sample of two thousand and four hundred increment cores.

The main results are as follows :

- mean values obtained for shrinkages with collapse are considerable: 72 p. 100. 28 p. $100,7,8$ p. 100 and 39 p. 100 for axial, tangential, radial and volumetric shrinkage, respectively. However retreatment with steam permits a recovery of 46 to 74 p. 100 of tangential shrinkage ;

- individual and infraspecific variability is very large for shrinkages and basic density. Volumetric shrinkage can vary, depending on the provenance, from 18 to 58 p. 100 , density from 443 to $593 \mathrm{~g} / \mathrm{dm}^{3}$ :

- a large and homogeneous region of provenances, the river Murray Basin, was thus identified : it is characterized by low densify, high increment and shrinkage :

- correlations between shrinkage and density are negative at individual and infraspecific level. Between provenance means, the correlation vigor with density is negative, between vigor and shrinkage positive ;

- the reputation of $E$. camaldulensis for not liking sites containing lime seems to be unjustified in view of the high vigor observed in Calabria. Besides a better growth the trees show on this site a higher density and less shrinkage than in Sicily ; studied.

- the interaction between genotype and environment appears to be weak for the characteristics

\section{Références bibliographiques}

ALLEGRET C., 1976. Le hêtre en Corrèze, D.D.A. de la Corrèze.

BORDEAUX Ch., 1977. Le hêtre de qualité dans le haut Causerans. Rapport ENITEF, O.N.F. St Girons. GREENHILL W. L., 1938. Collapse and its removal : some recent investigations with $E$, regnans. C.S.I.R. Division of Forest Products, Melbourne, Technical Paper, $n^{0} 24$.

HAYASHI, TERRAZAWA, 1977. Cell collapse under various conditions from an investigation of the effects on wood of drying, heating and other treatments. Wood Physics Laboratory, University of Nagoya, Japon.

JANIN G., 1973. Détermination des diagrammes polaires des rendements ef des indices de permanganate sur carottes droites et obliques. Essais de microclassage sur carottes obliques. Document de distribution limitée, Station de Recherches sur la Qualité des Bois, n० 1973/4.

JUINO Ph., 1977. Etude des relations entre vigueur et qualité du frêne et du merisier dans quelques stations du Nord-Est de la France. Rapport ENITEF, Station de Recherches sur la Qualité des Bois, C.N.R.F.

KANAGAWA, 1977. Influence of cell collapse on the shrinkage process of wood. University of Nagoyo, Japon.

KAUMAN W. G., 1960a. Contribution to the theory of cell collapse in wood. Investigations with Eucalyptus regnans. Australian Journal of Applied Science, vol. $11, \pi^{\circ} 1$. 
KAUMAN W. G., 1960b. Collapse in some Eucalyptus after treatment in inorganic salt solutions. Forest Products Journal, vol. $X, n^{\circ} 9$.

KAUMAN W. G., 1966. Cité par Polge, 1966.

KEYLWERTH R., 1954. Ein Beitrag zur qualifitiven Zuwagksanalyse. Holz als Roh- u. Werkstoff. vol. $12, \pi^{\circ} 3,77-83$.

LACAZE J. F., 1970. Etude de l'adaptation écologique des Eucalyptus. Compte rendu opérationnel. FAO, Rome.

NEPVEU G., 1973. Etude génétique de quelques qualités du bois de Pin maritime. Corrélations avec des caractères de croissance et d'aptitude à l'élagage. Document d̀ distribution limitée. Station de Recherches sur la Qualifé des Bois, C.N.R.F., no 1973/2.

NEPVEU G., 1976. Etude de l'anisotropie du bois dans une plantation de Pin laricio de Calabre de 12 ans. Document d̀ distribution limitée, Station de Recherches sur la Qualité des Bois. C.N.R.F., n $n^{\circ} 1976 / 4$.

NEPVEU G., KELLER R., TESSIER DU CROS E., 1978. Sélection juvénile pour la qualité du bois chez certains peupliers noirs. A paraitre dans Annales des Sciences Forestières, 35 (1).

POLGE H., 1966. Etablissement des courbes de variation de la densité du bois par exploration densitométrique des radiographies d'échantillons prélevés à la tarière sur des arbres vivants. Applications dans les domaines technologique et physiologique. Thèse de Docteur ès-Sciences Appliquées, Faculté des Sciences de I'Université de Nancy, 27 juin.

POLGE H., ILLY G., 1967. Observations sur l'anisotropie du pin maritime des Landes. Annales des Sciences Forestières, 24 (3).

POLGE H., 1973. Facteurs écologiques et qualité du bois. Annales des Sciences Forestières, 30 (2).

STAMM A. J., 1929. Density of wood substance, adsorption by wood and permeability of wood. Journal Agric. Res., 38, n० 1.

THIERCELIN F., POLGE H., 1970. Dégâts occasionnès par les sondages à la tarière, R.F.F., XXII, $n^{\circ} 6$, novembre-décembre.

THIERCELIN F., ARNOULD M. F., MANGENOT F., POLGE H., 1972. Altérations du bois provoquées par les sondages à la tarière. Leur contrôle. Annales des Sciences Forestières, 29 (1).

TIEMANN H. D., 1915. Cité par Greenhill, 1938.

THOBY M., 1975. Validité des tests précoces d'appréciation des caractères technologiques du bois de douglas. Application à une plantation comparative de vingt-quatre provenances de douglas (Peyrat-le-Château) en liaison avec des caractères morphologiques et auxométriques. Rapport ENITEF, Station de Recherches sur la Qualité des Bois, C.N.R.F.

VILLIĖRE A., 1966. Séchage du Bois. Dunod, Paris. 